\title{
Promise and challenge: Markers of prostate cancer detection, diagnosis and prognosis
}

\author{
D.A. Troyer ${ }^{\mathrm{a}, *}$, J. Mubiru ${ }^{\mathrm{a}}$, R.J. Leach ${ }^{\mathrm{b}}$ and S.L. Naylor ${ }^{\mathrm{b}}$ \\ ${ }^{a}$ Department of Pathology, University of Texas Health Science Center, San Antonio, TX, USA \\ ${ }^{\mathrm{b}}$ Cellular and Structural Biology, University of Texas Health Science Center, San Antonio, TX, USA
}

\begin{abstract}
Approximately 1 man in 6 will be diagnosed with prostate cancer during his life lifetime, and over 200,000 men in the U.S. are diagnosed with prostate cancer annually. Since the widespread adoption of PSA testing, about 60-70\% of men at risk in the U.S. have had a blood test for prostate cancer. With this, prostate cancer death rates have decreased, yet only slightly. Thirty thousand men still die each year from this disease. PSA testing fails to identify a small but significant proportion of aggressive cancers, and only about $30 \%$ of men with a "positive" PSA have a positive biopsy. Additionally, of men who are treated for prostate cancer, about $25 \%$ require additional treatment, presumably due to disease recurrence. Also of concern is the growing evidence that there are some prostate cancers for which treatment may not be necessary. Very long-term studies from the U.S. and Europe, following men with prostate cancer have found that some tumors do not progress over time. In these individuals, prostate cancer treatment is unnecessary and harmful as these men do not benefit from treatment but will be at risk of treatment-related side effects and complications. They suggest a fundamental problem with prostate cancer: it is not possible, at this time, to predict the natural history of the disease. It is for these reasons that the most important challenge in prostate cancer today is the inability to predict the behavior of an individual tumor in an individual patient. Here we review issues related to performance and validation of biomarkers with a focus on "doing no harm", and bearing in mind that it is the ultimate goal of early detection to save lives. Improved diagnostic and prognostic biomarkers are needed for prostate cancer, and the use of these markers should ultimately translate into increased life span and quality of life. The ultimate goal would be to not only have accurate biomarkers suitable for early diagnosis, but also biomarkers that identify men at greatest risk of developing aggressive disease. Technology has been brought to bear on this problem, and the major approaches are genomics, expression analysis, and proteomics. Proteomics and DNA methylation assays may soon be used in sensitive and specific diagnostic testing of serum and tissues for cancer. Expression arrays may be used to establish both a more specific diagnosis and prognosis for a particular tumor. The proteome is only beginning to be understood, and alternative splicing and post-translational modifications of proteins such as glycosylation and phosphorylation are challenging areas of study. Finally, risk assessment and prognosis are being pursued through analysis of genomic polymorphisms (single nucleotide polymorphisms, SNPs). This huge task is only beginning, and requires the combined expertise of molecular epidemiologists, oncologists, surgeons, pathologists, and basic scientists.
\end{abstract}

\section{Introduction}

The NCI Director's Challenge to the cancer research community is the elimination of suffering and death due to cancer by 2015 [1,2]. There is evidence that early diagnosis in the prostate specific antigen (PSA) "era" has

*Corresponding author: Dean Troyer, M.D., Professor, Dept. of Pathology, Mail Code 7750 , 7703 Floyd Curl Drive, San Antonio, TX, USA. Tel.: +1 210567 4101; Fax: +1 210567 2367; E-mail: Troyer@uthscsa.edu. led to a decline in death rates due to prostate cancer [3], and a decrease in the prevalence of advanced stage disease at the time of diagnosis [4]. However, there is continued disagreement over the efficacy of screening [5], the efficacy of PSA as a diagnostic marker [6], and the lack of effective treatments for advanced prostate cancer. Attitudes and policies concerning prostate cancer range widely from benign neglect [7] to advocacy of early detection [8]. It is possible that both views are correct based on the pathological $[9,10]$ and clinical $[11$, 12] heterogeneity of prostate cancer ranging from indolent, low grade disease to aggressive high grade disease 
which frequently metastasizes. Results of the recently published Prostate Cancer Prevention Trial affirm that prevention of prostate cancer can be achieved without undue side effects [5]. Future prevention efforts may also be tailored to high risk individuals with strategies ranging from dietary modification to pharmacological agents. A panel of biomarkers may therefore prove useful in more than one area of clinical decision making whether related to prevention, assessment of risk, diagnosis, or prognosis.

\section{Performance of diagnostic markers}

The goal is to develop diagnostic and prognostic markers useful in clinical decision making for diagnosis and treatment of prostate cancer. Early detection of early stage cancer has been recognized as an important goal in decreasing the morbidity and mortality of cancer [13]. However, there are important cautions that must be understood in the development and validation of assays for use in large scale screening of healthy populations. Investigators must consider the influence of sensitivity, specificity, and disease prevalence on the performance of a proposed biomarker, and to keep in mind that the ultimate goal of screening for cancer is reduction of mortality due to cancer [14]. Figure 1, shows the impact of changes in test specificity on the performance of a hypothetical screening assay with test sensitivity set at $100 \%$. Positive predictive value (PPV) is the probability that a subject has a disease when restricted to those individuals who test positive. PPV is calculated as follows: $\mathrm{PPV}=\mathrm{TP} /(\mathrm{TP}+\mathrm{FP})$ where $\mathrm{TP}$ and FP are the number of true positive and false positive results, respectively. A sharp decline in PPV can therefore result when the number of false positive cases increases due to a small decline in specificity. A decrease in specificity from 99.9 to $99 \%$ causes the positive predictive value (PPV) to decrease from 91 to $50 \%$ when disease prevalence is in the range of $1 \%$ which approximates the incidence of prostate cancer in an "at risk" population. This deterioration in positive predictive value may be ameliorated somewhat in practice if the primary screening test can be followed or confirmed with a second confirmatory test. Finally, the impact of a false positive test may vary according to the population and the disease. A significant false positive rate for mammographic screening for breast cancer occurs largely as a trade off for high sensitivity [15]. Importantly, definitive secondary tests (aspiration or biopsy) can be done under direct visualization to ascertain the

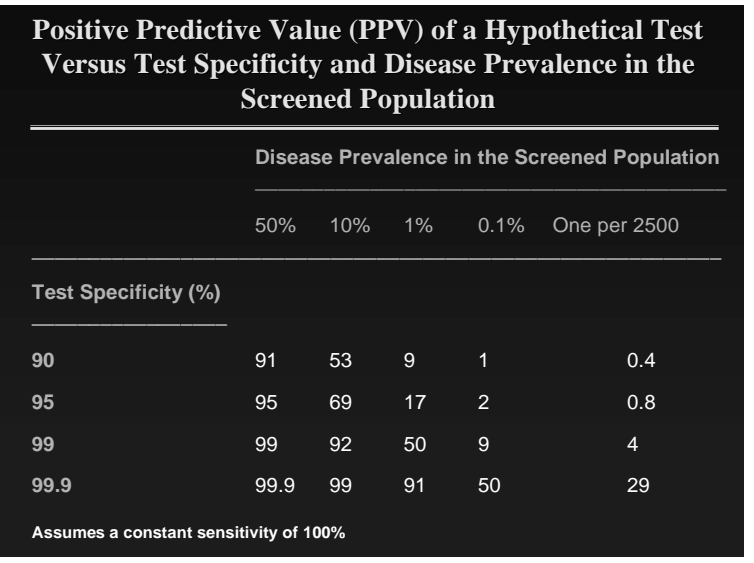

Fig. 1. Figure 1 shows the impact of changes in test specificity on the performance of a hypothetical screening assay with test sensitivity set at $100 \%$. Positive predictive value (PPV) is the probability that a subject has a disease when restricted to those individuals who test positive. PPV is calculated as follows: $\mathrm{PPV}=\mathrm{TP} /(\mathrm{TP}+\mathrm{FP})$ where TP and FP are the number of true positive and false positive results, respectively. (Courtesy of Dr. William Bigbee, U. of Pittsburgh).

nature of a palpable or mammographically abnormal breast lesion. Imaging technology has not progressed to this state in the prostate, so it is not feasible for the urologist to three-dimensionally target a specific lesion in the prostate.

\section{Marker validation}

A step-wise sequence of discovery, phased validation, and prospective clinical trials including psychosocial, ethical and economic assessment is recommended [16,17]. Pitfalls in moving from discovery to validation also include the nature of the (biologic) samples that are utilized in either discovery or early phase validation. The samples often tested in the discovery and early validation phases of a marker are those available ("at hand") to the investigator. Understandably, samples have often been collected retrospectively from subjects known to have cancer. These "available samples" can be helpful, but may lead to disappointment when the assay is performed in a more rigorous prospectively collected sample set in which age, ethnicity, and other variables are controlled. Even more importantly, a serum based marker which is discovered and initially validated in serum from subjects with advanced stage disease may prove disappointing when applied to subjects with early stage disease. Furthermore, it is helpful to recall that cancers morphologically evolve through pre-neoplastic, in-situ, and overtly 
malignant phases, and therefore, it is possible that certain biomarkers may be present both in morphologically malignant and morphologically benign epithelia. Highlighting this point is the recent demonstration that methylation events, which are proposed as biomarkers of cancer detection, occur in morphologically premalignant epithelium [18]. This demonstrates the feasibility of early detection using an assay which is potentially scalable to a high throughput platform. Utilization of this assay would require the understanding that not only dysplastic but even morphologically normal lesions may be detected. This is problematical because not all dysplastic lesions become cancer, and it will be necessary to understand the natural history of these "pre-malignant" lesions. The development of laser capture microscopy has enabled us to expand our understanding of genetic and epigenetic changes by allowing direct comparison of morphologically premalignant and malignant lesions [18].

Amongst the most desirable samples for early stage validation are "pre-diagnostic" serum or blood samples drawn at some interval prior to the clinical diagnosis of cancer. The assumption is that a biomarker assay which is truly effective in early diagnosis would perform robustly in this pre-clinical setting to diagnose early, curable disease. These samples are amongst the most difficult and expensive to obtain because they require prospective recruitment, screening, procurement and banking of samples from large numbers of healthy individuals. The costs ultimately embedded in such samples are high. Guidelines for the design of nested case control studies emphasize the need for combining samples from different sites and basing statistics on true positive and false positive rates rather than odds ratios or relative risks [19].

\subsection{Performance of PSA - state of the art}

Cancer statistics in the late 1990s reflect both a decline in prostate cancer mortality and "downstaging" of the disease at the time of diagnosis. Thus, the number of men who have metastases at the time of their initial diagnosis of prostate cancer has decreased in parallel with the decline in mortality [20]. While these favorable statistical trends followed the introduction of PSA as a screening assay for prostate cancer in the late 1980s and imply that earlier diagnosis and treatment provide benefit to the population, it is possible that PSA screening was part of an increased awareness of prostate cancer and heightened attention to the disease with more men being biopsied. Thus, while PSA is still recog- nized as the best available serum marker for detection of any form of cancer, there is substantial room for improvement. It is possible that the major effect of PSA may have been to stimulate biopsies.

The sensitivity of PSA as a screening assay for prostate cancer can be estimated from the incidence of cancer in the recently completed Prostate Cancer Prevention trial (PCPT) [5]. Of 7472 men biopsied at the end of study, 944 (13\%) had cancer. Positive cases were comprised of $10 \%$ of men on finasteride, (368/3652) and $15 \%$ of men on placebo (576/3820). $21 \%$ of men with positive end of study biopsies had PSA levels between 2.6 and $3.9 \mathrm{ng} / \mathrm{ml}$. $15.4 \%$ of the tumors found in men with PSA levels $<2.5 \mathrm{ng} / \mathrm{dl}$ were high grade tumors. In the PCPT trial setting therefore, the false negative rate for detection of high grade tumors (i.e. high grade tumors detected when PSA is $<$ $2.5 \mathrm{ng} / \mathrm{dl}$ ) is at least $15 \%$. The circumstances of the PCPT trial are unusual in that the likelihood is low that these men would have been biopsied in clinical practice. The false positive rate for PSA is more difficult to estimate, but available data [21] suggest that approximately $30 \%$ of men with an elevated PSA have a positive biopsy. There are ethnic variables which influence the performance of PSA. Only 25-35\% of Caucasians and Hispanics with a PSA in the $2-10 \mathrm{ng} / \mathrm{ml}$ range have biopsies positive for prostate cancer while up to $70-80 \%$ of African Americans with PSAs in this range have a positive biopsy [22,23]. Clearly, PSA sensitivity is suboptimal given a false negative rate approaching $15 \%$ and the specificity of PSA is also suboptimal since only about one third of men with an elevated PSA have a positive biopsy.

\subsection{Prostate cancer diagnosis - state of the art}

The gold standard for diagnosis of prostate cancer remains biopsy and pathological evaluation [24]. Core biopsies are difficult to interpret since the diagnosis of cancer is often based on a limited number of malignant glands amidst many benign glands. Basal cells are present in benign glands and can be identified by immunohistochemical staining for high molecular weight cytokeratin [34betaE12; keratin 903]. Using immunohistochemistry, alpha-methyl-CoA racemase (AMACR) has been shown to be highly overexpressed in prostate cancer [25]. Although produced by normal cells, the differentially increased expression of AMACR in cancer is such that up to $100 \%$ of prostate cancers stain by IHC for this enzyme with only rare staining of non-cancerous tissues in the prostate [25]. 
The staining characteristics of basal cells in benign glands can be exploited to support the diagnosis of cancer. $34 \beta \mathrm{E} 12$ and $\mathrm{p} 63$ have both been used to identify benign glands [26,27]. P63, a nuclear protein, shares homology with p53, and is selectively expressed in basal cells of the normal prostate, but is largely absent from prostatic adenocarcinoma [27]. It has been suggested that a cocktail of p63 and $34 \beta \mathrm{E} 12$ can improve the detection of benign basal cells vs. use of either alone [28]. Methylation of GSTpi, which appears to be nearly $100 \%$ sensitive in cancer, is another innovation which may be used to increase the sensitivity of the diagnosis of prostate cancer. It is consumptive of tissue, and this may be an impediment to its ubiquitous application [18].

\subsection{Diagnostic markers - new directions for proteins}

The ideal protein marker would be one which is specifically made by a tumor or its precursor morphologic lesion and which enters the circulation at an early enough stage to be a biomarker useful in the early detection of cancer. Curiously, PSA meets the second of these criteria since it does enter the circulation at an early stage, but it is not uniquely produced in tumor cells. Therein lies its relative weakness as a specific marker for cancer. Improved technology, including improved resolution and sample preparation methods, may enable both high throughput and specific identification of proteins by mass spectrometric techniques. The term proteomics is frequently applied to the combined application of mass spectroscopic analysis in tandem with other techniques for detection of proteins such as 2-D gel separation and microsequencing. Significant challenges intrinsic to the use of proteomic approaches to discover serum markers of cancer include detecting nanogram quantities of tumor-specific peptides amidst relatively massive (milligram) amounts of other serum proteins that are normally present [29].

There are two paradigms for use of this technology, one is pattern recognition, and the other is that the technology would be used to identify, isolate, and characterize unique proteins produced by cancer cells. The Ciphergen ProteinChip ${ }^{\circledR}$ SELDI ${ }^{\circledR}$-TOF-MS platform has been successfully used to discriminate serum protein profiles of patients with prostate cancer from case controls [30-32]. Because samples are directly applied to various matrices that are then subjected to displacement by laser energy, this approach has simplified sample handling. However, implementation of this as a screening tool will require will require synchronization of instruments, accounting for environmental effects on the matrixes, variability in proprietary elements in the matrices, and utilization of peak intensities for detection. Mass spectroscopy is extremely well suited to identification of peaks as a ratio of mass/energy, but peak heights are less accurate as a reflection of mass. This can create the need to develop appropriate statistical methods for selecting peaks. SELDI ${ }^{\circledR}$ may detect a cancer "phenotype" which when detected as a series of unique peaks, identifies the net effects of cancer on serum proteins. This cancer phenotype or profile may reflect the binding of small molecules to larger molecules, an altered profile of cytokines, altered binding of proteins to proteins, and altered or blocked cleavage sites [33]. The ability of SELDI ${ }^{\circledR}$ to distinguish the "profile" of serum from cancer patients from normal may reflect generalized cancer-induced alterations in the serum that are specific to cancer. However, it is still possible that none of the peaks detected at this level of resolution and sensitivity represent specific proteins made by tumors and secreted into the serum. The Early Detection Research Network is completing phased trials of this approach [34-36]. Approaches to develop appropriate peak selection alogorithms and statistical analysis of these patterns are being developed and tested [37]. A paradigm of using mass spectroscopy as a discovery tool for serum markers is also promising. When integrated with 2-D gels, it is possible to identify and characterize proteins specific to human tumors, an approach which is being applied to this problem $[38,39]$. Strategic approaches to the discovery process might integrate gene expression analysis with mass spectroscopy.

\section{Gene expression arrays}

Gene expression analysis has been expertly reviewed [40]. It exploits the affinity and binding of complementary cDNA to short corresponding nucleotide sequences (expressed sequence tags/ESTs) annealed to a substrate such as a glass slide. A fluorescence intensity signal is the read-out regardless of the platform, so the data consist of fluorescence intensity data. This allows up to approximately 10,000 ESTs to be evaluated on each slide. Most attention has focused on comparing tumor cDNA to normal cDNA, derived from the same subject. Overexpression of genes is confirmed by quantitative reverse transcriptase PCR techniques to verify that the RNA transcripts are actually elevated, and proteins, encoded by the overexpressed message, 
must also be characterized by immunohistochemistry or other means. Gene expression data can be seen as a tool to be used for annotating the expression profiles of tumors. Even without reference to normal DNA, analysis of gene expression array data can be used to independently categorize tumors [41]. This has the potential to supplement the morphologic diagnosis and classification of tumors, and may provide important prognostic information. Expression array data, like proteomic data, can be used both in "pattern recognition", and also as a discovery tool to identify specific proteins overexpressed in cancer. The classification of tumors using array data falls in some measure into the "pattern recognition" paradigm utilized in analysis of SELDI ${ }^{\circledR}$ data. However, expression array analysis is more technically demanding and costly than. SELDI ${ }^{\circledR}$. The costs and technical complexities of expression array data are balanced by the fact that, unlike the peaks observed in SELDI, each oligonucleotide on the array can be linked to a specific target (gene). Even so, expression arrays do not reveal the entire spectrum of expressed proteins. Alternate splicing of mRNA transcripts occurs, a point worth noting since one gene may give rise to several different transcripts, expanding by several-fold the number of distinct mRNAs [42]. Obviously, both over- and underexpression of genes has implications for the underlying biology of cancer as well. Underexpression of genes is frequently linked to methylation events, and may thereby reflect down regulation of gene expression [43]. Overexpression of genes observed in prostate cancer include AMACR and hepsin, both of which can be overexpressed thirty to forty fold above normal control levels [44-46]. Recent studies using RT-PCR and immunohistochemistry have confirmed the overexpression of hepsin [47] and AMACR [48]. AMACR is a mitochondrial and peroxisomal enzyme which, through conversion of (2R)stereoisomers of phytanic and pristanic acid to their (S) counterparts is essential in the $\beta$-oxidation of bile acid intermediates, branched-chain fatty acids and for degradation of bile acid intermediates such as di-and trihydroxycholestanoic acid [49]. AMACR has been recently shown to function as a growth promoter which functions independently of androgens [50]. Hepsin has also been recently shown to be involved in regulation of cell growth [51]. An alternately spliced variant of the AMACR family was subsequently discovered "in silico" [52], and further studies demonstrated more members of this family [53]. These are examples of how the discovery process has been accelerated through the application of high throughput technology. While it is unlikely that expression arrays comprised of thousands of ESTs will be used in a clinical setting, the technology promises to accelerate the discovery of over- and underexpressed proteins and "focused" arrays may be useful in assaying diagnostic and prognostic biomarkers. The classification of tumors using expression arrays [41] may provide important prognostic data however, and it is possible that this will become a standard part of the clinical/diagnostic armamentarium, especially if specific chips are produced that target specific tumors with a limited number of genes.

\section{Risk assessment}

Markers of risk for any disease can be beneficial in leading to early diagnosis and potential cure or prevention of disease. Prevention strategies may be targeted to those at highest risk, avoiding exposure of the entire population, and thereby diminishing the potential complications associated with the intervention. Effective markers of risk allow for refinement of screening programs targeting early detection of cancer. The dictum "do no harm" is especially relevant when large numbers of healthy individuals are screened for cancer. Even in the case of ovarian cancer with sensitivity and specificity well above $90 \%$, frequent screening of healthy populations increases the risk of adverse outcomes due to false positive results, unnecessary additional testing and procedures, overdiagnosis of cancer, and anxiety [54]. The ability to identify individuals at high risk for disease would permit less frequent screening in men at lower risk of prostate cancer and concomitantly decreasing the false positive rate. The goal is to increase the specificity of the screening and reduce the frequency of biopsies.

The search for markers of risk may be directed by hypotheses regarding causes of prostate cancer. Age, diet, and genetic factors have all been implicated in the risk of prostate cancer. The incidence of prostate cancer rises sharply in the fifth and six decades of life, about a decade later than the pattern observed for breast cancer [11]. The major tumors of adults including prostate, breast, colon, and lung cancer, thus all bear some relationship to aging, and cumulative effects of free radicals [55,56] and DNA mis-match repair [57] are invoked as potential causes.

The striking geographical differences in the incidence of prostate cancer, and the fact that incidence rates for immigrant populations tend to revert to those of the host country have stimulated interest in diet and 
environmental risks. Although these observations support the hypothesis that environmental and dietary factors account for some of the risks of prostate cancer, these factors have not been identified [58]. Dietary risk factors that have been examined for their impact on prostate cancer include macronutrient intake (fats, carbohydrates, and protein) $[59,60]$ micronutrient intake [61,62] and overnutrition [63]. Pre-clinical and clinical data, derived in some cases from secondary analyses of population-based epidemiologic studies designed for other end-points, has provided evidence that a variety of micronutrients and pharmacologic agents are related to prostate cancer $[61,62]$. It is probable that nutritional factors, aging, and genetics all influence the pathogenesis of prostate cancer and are therefore logical places to look for specific indicators of risk. One of the difficulties in studying oxidative damage has been the lack of a reliable assay to study DNA damage, and it is intriguing to note that recent studies suggest that expression of mRNA for base excision repair genes may reflect DNA damage (OGG1, APE, MPG, and Pol $\beta$ ), whereas no effect was observed on DNA repair genes that were not related to oxidative DNA damage (e.g., $O^{6}$-methylguanine methyl transferase, MGMT) [64].

\section{Genetic factors in prostate cancer}

A recent study of twins estimated [65] that 42 percent of the risk for prostate cancer was explained by hereditary components. This compared to only 35 percent for colorectal carcinoma and 27 percent for breast cancer. Somatic changes in prostate cancers include deletions at 8p, 10q, 13q, 16q, 18q, and $Y$ and gains at $7 p, 7 q, 8 q, X q[66-68]$. The frequency of these somatic gains or deletions increases with the stage of disease. However, inherited variants may be important in prostate cancer, and large scale mapping methods that proved successful for breast and colon cancer have been utilized to search for high penetrance susceptibility genes. Genes identified to date include HPC1 at 1q24 [69], ELAC2 at 17p11 [70], MSR1 8p22-23 [71], $P C A P$ at $1 \mathrm{q} 42, H P C X$ at $\mathrm{Xq} 27, C A P B$ at $1 \mathrm{p} 36, H P C 20$ at $20 \mathrm{q} 13$, and [72]. These genes may play a role in both familial and sporadic prostate cancer; however, their role in the general population may be minor [73]. It has been hypothesized that the major contribution of heritable factors in prostate and other cancers may be attributed to common polymorphisms in prostaterelated genes rather than mutations in high penetrance susceptibility genes [74]. Combinations of these weak genetic variants would be hypothesized to have a much greater impact on public health than would the familial genes [75]. While single alterations in prostaterelated genes may only modestly increase the risk of cancer, the collective impact of these polymorphisms on cancer incidence may be greater than the familial high-penetrance susceptibility genes. Polymorphisms are genetic alterations in coding and non-coding regions of DNA such as length polymorphisms or single nucleic acid substitutions. Whether collectively related to cause or simply linked to other genes that cause cancer, these polymorphisms can be used as markers of risk [76]. In some regions, polymorphisms can be grouped by haplotypes which are several or more polymorphisms that are inherited in blocks [76]. Once the status of one of the polymorphisms is known, the others are also known. Haplotypes, with their constituent polymorphisms may be viewed as markers of risk. An important feature of the distribution of this risk of disease in populations, including prognostic or risk factors for specific cancers, is that it is not uniformly distributed throughout the population. Thus, a corollary of the polygenic model of risk of cancer is that risk is concentrated in a relatively small proportion of the population [75]. If breast cancer can be used to inform our approach to prostate cancer, the risk of disease is predicted to vary as much as 40 -fold higher in the $1 / 5$ of the population at high risk vs. the $1 / 5$ of the population at lowest risk. Thus, it is estimated that about one half of all breast cancers occur in a subset of women whose risk of developing breast cancer before the age of 70 is $10 \%$. In contrast, only $12 \%$ of all breast cancers occur in a lower risk subpopulati on whose risk of developing cancer before the age of 70 is $3 \%$ [75]. This is the scenario that would be most favorable to guiding large scale screening in healthy populations. Also underlying the polygenic hypothesis is the prediction that polymorphisms which underlie cancers (and other polygenic diseases) derive from common variants that arose once, early in the history of human evolution [77]. It is anticipated that the search for genomic biomarkers of prostate cancer will be aided by projects such as the SNP consortium (http://snp.cshl.org), the Haplotype Map (HapMap) Project being organized by the National Institutes of Health, and The International HapMap Project launched by the National Human Genome Research Institute. This three year project will place segments of genetic code that are inherited as a group (haplotype) into a database. The long term goal is to link haplotypes to specific disease states. 


\section{Epigenetic modifications}

Methylation of DNA and histones may be useful diagnostic markers [78]. Methylation of both DNA and histones occurs, and both play a role in gene function and expression. In addition, phosphorylation and acetylation of the N-terminal tails of histones occurs [79]. Methylation of $\mathrm{CpG}$ islands in DNA is of interest for both mechanistic and prognostic/diagnostic reasons. Methylation of one or both alleles of a region can serve as a biomarker of cancer or silence gene expression when they are in a promoter region [78]. Long understood as important modulaters of gene expression during development, assays for methylation are appealing to translational research, since they can be based on amplification techniques, and thereby utilize small amounts of biologics. This is particularly advantageous when small amounts of sample are available. Methylation of $\mathrm{CpG}$ islands in the promoter of the pi class of glutathione-S-transferase (GSTpi) occurs in prostatic intraepithelial neoplasia (PIN) [80], proliferative inflammatory atrophy (PIA) and cancer [80]. Other hypermethylated regions relevant to prostate cancer include the retinoic acid receptor beta 2 [81]. These findings in prostate cancer suggest that DNA methylation is among the early events in tumorigenesis, but it remains to be seen whether DNA methylation is a necessary or permissive event in tumorigenesis. Methylation of GSTpi is highly specific and sensitive as a diagnostic tool [82], and several assays are under commercial development. It remains to be clarified when GSTpi methylation occurs during carcinogenesis.

\section{Markers of prognosis}

We know from epidemiological studies that only a minority of patients with the histological diagnosis of prostate cancer develop clinically significant and lifethreatening tumors, and that many patients die (from other causes) with an indolent form of prostate cancer [11]. This has engendered use of the terms "clinical" and "histological" to specify the existence of prostate cancers with varying outcomes. The terms are not commonly used in clinical practice, but they allude to an awareness that a substantial majority of elderly autopsied males will have prostatic adenocarcinoma, but that this is not clinically significant disease. Epidemiological evidence supports the concept that patients with histological evidence of prostate cancer have a wide range of outcomes. This indicates that not all "histological tumors" are equivalent, and that adenocarcinoma of the prostate is a heterogeneous disease. In spite of the addition of PSA and transrectal ultrasound as diagnostic too ls, we still lack prospective criteria which would define the terms clinically signifcant vs. histological or indolent tumors. The dilemma of which prostate tumors should be treated aggressively vs. those which may bear watchful waiting remains unresolved.

The material available for assessment of prognosis may be blood or tissue from a biopsy or prostatectomy. For patients electing non-surgical treatment of disease, biopsy would be the only tissue available for assessment of prognosis. The mainstays used in clinical decision making are pre-treatment PSA levels, clinical stage by digital rectal exam, and Gleason's score on needle biopsy [83]. Increasingly, there is recognition that up to 10 prostate biopsy cores may be appropriate, and that the cores should specifically sample the apex and lateral regions of the posterior prostate [84]. Involvement of multiple systematically sampled biopsy cores by tumor is predictive of adverse pathological findings at radical prostatectomy, but a small amount of tumor in one core doesn't predict favorable pathological staging. Gleason's grade is a powerful predictor of outcome, correlating with pathological variables seen in radical prostatectomy specimens.

Markers derived from pathological staging of prostatectomies include Gleason's grade, margin status, spread outside the capsule (extraprostatic extension), and seminal vesicle invasion. Lymph node excision is performed infrequently because the incidence of positive nodes in patients coming to prostatectomy is less than $5 \%$. Low risk subjects are those who at prostatectomy have a Gleason's score of $\leqslant 3+3=6$ with negative surgical margins. $95 \%$ of these men have no evidence of disease at 10 years. Risk of recurrence of disease at 10 years increases to approximately $28 \%$ with either a Gleason's score of $7 / 10$ and negative surgical margins or a Gleason's score of $6 / 10$ and positive surgical margins. When seminal vesicle invasion is present, or Gleason's score is $7 / 10$ with a positive surgical margin or Gleason's score is $\geqslant 8 / 10$, the chance of disease recurrence at 10 years rises to approximately $60 \%$. When pelvic lymph nodes are positive, the chances of disease recurrence rise to nearly $90 \%$ [85].

While these pathological features are predictive of disease recurrence, they do not prospectively distinguish between aggressive and "indolent" tumors in an individual patient nor do they predict response to neoadjuvant therapy. Thus, it is not clear that prostate- 
ctomy is necessary in all early stage cancers; however, in the absence of useful markers that predict biological behavior, men with both aggressive and indolent tumors are subjected to prostatectomy. A serum marker would be ideal for prognostic purposes. In a recent single institution analysis of over 700 prostatectomy cases, pretreatment plasma levels of interleukin-6 soluble receptor (IL6SR) and transforming growth factor beta1 (TGF-beta1) were useful adjuncts in predicting progression of disease [86].

The development of prognostic markers is complicated by the frequent existence of multiple independent primary tumors in the prostate [87]. Multiple primary tumors are occasionally observed in other organs such as breast and colon, but most often in association with rare cancer susceptability genes (e.g., polyposis coli) or with metachronous development of subsequent tumors. However, the vast majority of breast, colon, and lung tumors are resected and treated as single tumors. In contrast, histopathological examination of prostatectomies frequently reveals the presence of multiple, independent tumors, and this has now been confirmed by genetic studies [88]. This observation leads, in turn, to the question "Do independent tumors in the prostate have equivalent potential for invasion and metastasis, or are they biologically different?" Serial examinations of whole mount sections of prostate provided the first evidence that multiple tumors can be identified in the prostate [89]. More recent studies suggest that the features of the "index" tumor in a prostatectomy accurately predict the likelihood of recurrence and that this is largely independent of the smaller, concurrent tumors [87].

\section{Future directions and strategies}

The detection of antibodies to tumor antigens is a potential means to discover biomarkers. Tumor derived antigens that elicit a humoral response can result from overexpression of normal or mutated genes [90]. This antibody response is quite heterogeneous, but in combination, multiple antibodies to such tumor antigens may be useful in cancer diagnosis [91]. One promising approach to identification of tumor antigens is through serological identification of tumor antigens by recombinant expression cloning (SEREX) [92,93]. This technique involves screening for tumor antigens using IgG rich sera from cancer patients against an expression library derived from the RNA of the autologous tumor. Since the technique identifies cancer specific antigens, and is based on antibody mediated recognition, it is both sensitive and specific. Mintz et al. recently identified a consensus motif in prostate cancer using phage display to detect peptides recognized by circulating tumor antibodies [94]. Disadvantages of SEREX are that post-translational modifications of proteins are not accounted for since the targets are expressed in prokaryotic systems and it is difficult to scan large numbers of tumors [95]. An alternative approach is screening of 2-D gels with patient sera to identify proteins which have elicited an antibody response [96]. Proteins that elicit an antibody response are identified by mass spectroscopy [96]. In another platform, somewhat reversing the SEREX strategy, antibodies of known specificity are annealed to a substrate to create a microarray capable of screening sera in a high throughput manner [97]. This platform may be particularly helpful in monitoring post-translational modifications such as phosphorylation.

Advances in mass spectroscopy are also promising. Sample preparation is one of the challenges of incorporating mass spectroscopy into high throughput translational research. It is particularly frustrating that some of the most interesting proteins such as integral membrane proteins and glycoproteins are the most difficult to extract and prepare for mass spectroscopy [98]. Integral membrane proteins have both hydrophilic and hydrophobic regions conferred by the membrane-spanning, and intra- and extracellular domains. They are difficult to solubilize and are underrepresented in proteomic analyses even though it is estimated that $20-30 \%$ of all open reading frames encode for integral membrane proteins [99]. These proteins have proven challenging even for gel electrophoresis [99]. Avidin-affinity purification of biotinylated proteins has been combined with microcapillary reversed-phase HPLC tandem mass spectrometry to overcome some of these problems [99]. High $\mathrm{pH}$ combined with proteinase digestion has allowed for improvements in sample preparation that may further enhance the ability to characterize the membrane proteome [100]. Similarly, glycosylated proteins have been difficult to study, but are likely to be relevant to the unique signature of cancers since a universal hallmark of cancer cells is an alteration in their glycosylation phenotype [101]. The incorporation of lectin binding into sample preparation can facilitate mass spectroscopic analysis of glycosylated proteins [102] offering promise for future developments in this area. Finally, the integration of proteomics and genomics has identified promising prognostic biomarkers in lung cancer, highlighting the importance of using multiple platforms to discover and validate biomarkers [103]. 


\section{Summary and conclusion}

Data currently accessible for clinical decision making include serum prostate specific antigen (PSA) levels, age, race, and Gleason's grade of a biopsied tumor. To this, in the future, may be added data from genetic and proteomic analyses of individual tumors developed in the context of imaging or mapping technology which describe the size and location of tumors in vivo. While PSA is a widely used serum marker of cancer, there is room for improvement. On the near horizon for use as diagnostic and prognostic biomarkers are methylation assays, gene expression arrays, and mass spectroscopy, including SELDI ${ }^{\circledR}$. Integration of these approaches during the discovery process will likely produce biomarkers useful in clinical decision making [104]. As the sensitivity and specificity of molecular markers improves, the likelihood will increase that biomarkers can provide diagnostic and prognostic data which function independently of clinical and imaging data $[105,106]$. On the more distant horizon is the exciting prospect of profiling large populations for risk using genetic polymorphisms. This would reduce the number of annual screenings, target prevention strategies to those most likely to be affected, and likely provide prognostic information as well. It is exciting and intimidating to realize that the real difficulty may be in selecting a subset of biomarkers from the numerous existing and emerging prospects for validation and application in a clinical setting.

\section{Acknowledgements}

Support of the National Institutes of Health grant 5U01-CA-86402-02, the Early Detection Research Network; The Presidential Research Enhancement Fund from the University of Texas Health Science Center, San Antonio; an Institutional Research Grant from the University of Texas Health Science Center San Antonio are gratefully acknowledged; and the American Cancer Society are gratefully acknowledged

\section{References}

[1] The Cancer Letter 29, page 5.

[2] BenchMarks 3, issue 2 http://cancer.gov/BenchMarks/about. html.

[3] J.L. Stanford, R.A. Stephenson, L.M. Coyle et al., Prostate Cancer Trends 1973-1995, SEER Program, National Cancer Institute. NIH Pub. No 99-4543, Bethesda, MD, 1999.
[4] D.C. Miller, K.S. Hafez, A. Stewart, J.E. Montie and J.T. Wei, Prostate carcinoma presentation, diagnosis, and staging: an update form the National Cancer Data Base, Cancer 98 (2003), 1169-1178.

[5] I.M. Thompson, P.J. Goodman, C.M. Tangen et al., The Influence of Finasteride on the Development of Prostate Cancer, New Eng J Med 349 (2003), 213-222.

[6] I.M. Thompson, R.J. Leach, B.H. Pollock and S.B. Naylor, prostate cancer and prostate-specific antigen: The more we know, the less we understand, J Nat Can Inst 95 (2003), 1027-1028.

[7] A.J. Costello, Contemporary view of prostate cancer diagnosis and treatment, Australian Family Physician 32 (2003), 396-398.

[8] R.M. Merrill and R.A. Stephenson, Trends in mortality rates in patients with prostate cancer during the era of prostate specific antigen screening, J Urology 163 (2000), 503-510.

[9] D.R. Greene, E. Rogers, E.C. Wessels, T.M. Wheeler, S.R. Taylor, R.A. Santucci, T.C. Thompson and P.T. Scardino, Some small prostate cancers are nondiploid by nuclear image anslysis: Correlation of deoxyribonucleic acid ploidy status and pathological features, J. Urology 151 (1994), 1301-1307.

[10] T. Saric, Z. Brkanac, D.A. Troyer, S.S. Padalecki, M. Sarosdy, K. Williams, L. Abadesco, R.J. Leach and P. O'Connell, Genetic pattern of prostate cancer progression, Int J Cancer 81 (1999), 219-224.

[11] E.D. Crawford, Epidemiology of Prostate Cancer, Urology 62(6 Suppl 1) (2003), 3-12.

[12] O.W. Brawley, K. Knopf and I. Thompson, The epidemiology of prostate cancer part II: the risk factors, Semin Urol Oncol 16 (1998), 193-201.

[13] R. Etzioni, N. Urban, S. Ramsey, M. McIntosh, S. Schwartz, B. Reid, J. Radich, G. Anderson and L. Hartwell, The case for early detection, Nat Rev Cancer 3 (2003), 243-252.

[14] B.S. Kramer, General Keynote: Cancer Screening: Translation of Principles into Practice, Gynecol Oncol 88 (2003), S71-S74.

[15] E. Silverman, S. Woloshin, L.M. Schwartz, S.J. Bryam, H.G. Welch and B. Fischoff, Women's views on breast cancer risk and screening mammography: a qualitative interview study, Medical Decision Making 21 (2001), 231-240.

[16] M.S. Pepe, R. Etzioni, Z. Feng, J.D. Potter, M.L. Thompson, M. Thornquist, M. Winget and Y. Yasui, Phases of Biomarker Development for Early Detection of Cancer, J. Nat.Cancer Institute 93 (2001), 1054-1061.

[17] P.E. Barker, Cancer biomarker validation: standards and process: roles for the National Institute of Standards and Technology (NIST), Annals of the New York Academy of Sciences 983 (2003), 142-150.

[18] M. Nakayama, C.J. Bennett, J.L. Hicks, J.I. Epstein, E.A. Platz, W.G. Nelson and A.M. De Marzo, Hypermethylation of the human glutathione S-transferase-pi gene (GSTP1) $\mathrm{CpG}$ island is present in a subset of proliferative inflammatory atrophy lesions but not in normal or hyperplastic epithelium of the prostate: a detailed study using laser-capture microdissection, Am J Path 163 (2003), 923-933.

[19] S.G. Baker, B.S. Kramer and S. Srivastava, Markers for early detection of cancer: statistical guidelines for nested case-control studies, BMC Medical Research Methodology 2 (2002), 4.

[20] C.J. Mettlin, G.P. Murphy, D.S. Rosenthal and H.R. Menck, The National Cancer Data Base report on prostate carcinoma after the peak in incidence rates in the U.S. The American 
College of Surgeons Commission on Cancer and the American Cancer Society, Cancer 83 (1998), 1679-1684.

[21] R.J. Babaian, D.A. Johnston, W. Naccarato et al., The incidence of prostate cancer in a screening population with a serum prostate specific antigen between 2.5 and $4.0 \mathrm{ng} / \mathrm{ml}$ : relation to biopsy strategy, J Urol 165 (2001), 757-760.

[22] J.S. Lam, Y.K. Cheung, M.C. Benson and E.T. Goluboff, Comparison of the predictive accuracy of serum prostate specific antigen levels and prostate specific antigen density in the detection of prostate cancer in Hispanic-American and white men, J Urology 17 (2003), 451-456.

[23] I. Abdalla, P. Ray, F. Vaida and S. Vijayakumar, Racial differences in prostate-specific antigen levels and prostate-specific antigen densities in patients with prostate cancer, American Journal of Clinical Oncology 22 (1999), 537-541.

[24] A.M. DeMarzo, W.G. Nelson, W.B. Isaacs and J.I. Epstein, Pathological and molecular aspects of prostate cancer, Lancet 361 (2003), 955-964.

[25] C. Magi-Galluzzi, J. Luo, W.B. Isaacs, J.L. Hicks, A.M. de Marzo and J.I. Epstein, Alpha-methylacyl-CoA racemase: a variably sensitive immunohistochemical marker for the diagnosis of small prostate cancer foci on needle biopsy, American Journal of Surgical Pathology 27 (2003), 1128-1133.

[26] C. Magi-Galluzzi, J. Luo, W.B. Isaacs, J.L. Hicks, A.M. de Marzo and J.I. Epstein, Alpha-methylacyl-CoA racemase: a variably sensitive immunohistochemical marker for the diagnosis of small prostate cancer foci on needle biopsy, American Journal of Surgical Pathology 27 (2003), 1128-3.

[27] R.B. Shah, M. Zhou, M. LeBlanc, M. Snyder and M.A. Rubin, Comparison of the Basal Cell-Specific Markers, 34[beta]E12 and p63, in the Diagnosis of Prostate Cancer, Am J Surg Path 26 (2002), 1161-1168.

[28] M. Zhou, R. Shah, R. Shen and M.A. Rubin, Basal cell cocktail (34betaE12 + p63) improves the detection of prostate basal cells, American Journal of Surgical Pathology 27 (2003), 365-371.

[29] E.P. Diamandis, Point: Proteomic patterns in biological fluids: do they represent the future of cancer diagnostics? Clinical Chemistry 49 (2003), 1272-1275.

[30] B.-L. Adam, Y. Qu, J.W. Davies, M.D. Ward, M.A. Clements, L.H. Cazares et al., Serum protein fingerprinting coupled with a pattern-matching algorithm distinguishes prostate cancer from benign prostate hyperplasia and healthy men, Cancer Res 62 (2002), 3609-3614.

[31] E.F. Petricoin, III, D.K. Ornstein, C.P. Paweletz, A. Ardekani, P.S. Hackett, B.A. Hitt et al., Serum proteomic patterns for detection of prostate cancer, J Natl Cancer Inst 94 (2002), 1576-1578

[32] Y. Qu, B.-L. Adam, Y. Yasui, M.D. Ward, L.H. Cazares, P.F. Schellhammer et al., Boosted decision tree analysis of surface-enhanced laser desorption/ionization mass spectral serum profiles discriminated prostate cancer from nonprostate patients, Clin Chem 48 (2002), 1835-1843.

[33] L.A. Liotta, M. Ferrari and E. Petricoin, Clinical proteomics: written in blood, Nature 425 (2003), 905.

[34] B.L. Adam, Y. Qu, J.W. Davis, M.D. Ward, M.A. Clements, L.H. Cazares, O.J. Semmes, P.F. Schellhammer, Y. Yasui, Z. Feng and G.L. Wright Jr., Serum protein fingerprinting coupled with a pattern-matching algorithm distinguishes prostate cancer from benign prostate hyperplasia and healthy men, Cancer Research 62 (2002), 3609-3014.

[35] T. Reynolds, Validating biomarkers: early detection research network launches first phase III study, J Natl Cancer Inst 95 (2003), 422-423.
[36] W.E. Grizzle, O.J. Semmes, J. Basler, E. Izbicka, Z. Feng, J. Kagan, B.-L. Adam, D. Troyer, S. Srivastava, M. Thornquist, Z. Zhang and I.M. Thompson, The early detection research network surface-enhanced laser desorption and ionization prostate cancer detection study. A study in biomarker validation in genitourinary oncology, Urologic Oncology, in press.

[37] Y. Yasui, M. Pepe, M.L. Thompson, B.L. Adam, G.L. Wright, Jr., Y. Qu, J.D. Potter, M. Winget, M. Thornquist and Z. Feng, A data-analytic strategy for protein biomarker discovery: profiling of high-dimensional proteomic data for cancer detection, Biostatistics 4 (2003), 449-463.

[38] J. Madoz-Gurpide and S.M. Hanash, Molecular analysis of cancer using DNA and protein microarrays, Advances in Experimental Medicine \& Biology 532 (2003), 51-58.

[39] A.A. Alaiya, M. Oppermann, J. Langridge, U. Roblick, L. Egevad, S. Brindstedt, M. Hellstrom, S. Linder, T. Bergman, H. Jornvall and G. Auer, Identification of proteins in human prostate tumor material by two-dimensional gel electrophoresis and mass spectrometry, Cellular \& Molecular Life Sciences 58 (2001), 307-311.

[40] J. Luo, W.B. Isaacs, J.M. Trent and D.J. Duggan, Looking beyond morphology: cancer gene expression profiling using DNA microarrays, Cancer Investigation 21 (2003), 937-949.

[41] T. Sørlie, R. Tibshirani, J. Parker, T. Hastie, J.S. Marron, A. Nobel, S. Deng_, H. Johnsen, R. Pesich, S. Geisler, J. Demeter, C.M. Perou, P.E. Lønning and P.O. Brown, AnneLise B?rresen-Dale, and D. Botstein, Repeated observation of breast tumor subtypes in independent gene expression data sets, Proc. Nat. Acad. Sci. USA 100 (2003), 8418-8423.

[42] S. Stamm, Signals and their transduction pathways regulating alternative splicing: a new dimension of the human genome, Human Mol Genetics 11 (2002), 2409-2416.

[43] W.H. Lee, R.A. Morton, J.I. Epstein, J.D. Brooks, P.A. Campbell, G.A. Bova, W.S. Hsieh, W.B. Issacs and W.G. Nelson, Cytidine methylation of regulatory sequences near the -class glutathione S-transferase gene accompanies human prostatic carcinogenesis, Proc Natl Acad Sci USA 91 (1994), 1173311737.

[44] S.M. Dhanasekaran, T.R. Barrette, D. Ghosh, R. Shah, S. Varambally, K. Kurachi et al., Delineation of prognostic biomarkers in prostate cancer, Nature 412 (2001), 822-.

[45] J.A. Magee, T. Araki, S. Patil, T. Ehrig, L. True, P.A. Humphrey et al., Expression profiling reveals hepsin overexpression in prostate cancer, Cancer Res 61 (2001), 5692-.

[46] J.B. Welsh, L.M. Sapinoso, A.I. Su, S.G. Kern, J. WangRodriguez, C.A. Moskaluk et al., Analysis of gene expression identifies candidate markers and pharmacological targets in prostate cancer, Cancer Res 61 (2001), 5974-.

[47] C. Stephan, G.M. Yousef, A. Scorilas, K. Jung, M. Jung, G. Kristiansen, S. Hauptmann, T. Kishi, T. Nakamura, S.A. Loening and E.P. Diamandis, Hepsin is highly over expressed in and a new candidate for a prognostic indicator in prostate cancer, Journal of Urology 171 (2004), 187-191.

[48] Z. Jiang, B.A. Woda, K.L. Rock, Y. Xu, L. Savas, A. Khan, G. Pihan, F. Cai, J.S. Bacook, P. Rathanaswami, S.G. Reed, J. Xu and G.R. Fanger, P504S A new molecular marker for the detection of prostate carcinoma, Am. J. Surg. Pathol 25 (2001), 1397-1404.

[49] S. Ferdinandusse, S. Denis, P.T. Clayton, A. Graham, J.E. Rees, J.T. Allen, B.N. McLean, A.Y. Brown, P. Vreken, H.R. Waterham and J.A. Wanders, Mutations in the gene encoding peroxisomal á-methylacyl-CoA racemase cause adult-onset 
sensory motor neuropathy, Nature Genetics $\mathbf{2 4}$ (2000), 188191.

[50] S. Zha, S. Ferdinandusse, S. Denis, R.J. Wanders, C.M. Ewing, J. Luo, A.M. De Marzo and W.B. Isaacs, Alphamethylacyl-CoA racemase as an androgen-independent growth modifier in prostate cancer, Cancer Research $\mathbf{6 3}$ (2003), 7365-7376.

[51] V. Srikantan, M. Valladares, J.S. Rhim, J.W. Moul and S. Srivastava, Hepsin inhibits cell growth/invasion in prostate cancer cells, Cancer Research 62 (2002), 6812-6816.

[52] G.L. Shen-Ong, Y. Feng and D.A. Troyer, Expression Profiling Identifies a Novel á- Methylacyl-CoA Racemase Exon with Fumarate Hydratase Homology, Cancer Research 63 (2003), 3296-3301

[53] J.N. Mubiru, G.L. Shen-Ong, A.J. Valente and D.A. Troyer, Alternative Spliced Variants of the Alpha-methyl-acyl-CoA Racemase Gene and their Expression in Prostate Cancer, Gene 327 (2004), 89-98.

[54] U. Menon and I. Jacobs, Screening for ovarian cancer, Best Practice \& Research in Clinical Obstetrics \& Gynaecology 16 (2002), 469-482.

[55] N. Caporaso, The molecular epidemiology of oxidative damage to DNA and cancer, Journal of the National Cancer Institute 95 (2003), 1263-1265.

[56] H. Van Remmen, M.L. Hamilton and A. Richardson, Oxidative damage to DNA and aging, Exercise \& Sport Sciences Reviews 31 (2003), 49-53.

[57] R.D. Wood, M. Mitchell, J. Sgouros and T. Lindahl, Human DNA repair genes, Science 291 (2001), 1284-1289.

[58] D.S. Coffey, Similarities of prostate and breast cancer: Evolution, diet, and estrogens, Urology 57 (2001), 31-38.

[59] R.L. Prentice and L. Sheppard, Dietary fat and cancer: consistency of the epidemiological data, and disease prevention that may follow from a practical reduction in fat consumption, Cancer Causes Control 1 (1990), 81-97.

[60] J. Satia-About a, R.E. Patterson, R.N. Schiller and A.R. Kristal, Energy from fat is associated with obesity in U.S. men: results from the Prostate Cancer Prevention Trial, Preventive Medicine 34 (2002), 493-501.

[61] M.S. Cooke, M.D. Evans, M. Dizdaroglu and J. Lunec, Oxidative DNA damage: mechanisms, mutation, and disease, FASEB J 17 (2003), 1195-1214.

[62] G.E. Goodman, S. Schaffer, G.S. Omenn, C. Chen and I. King, The association between lung and prostate cancer risk, and serum micronutrients: results and lessons learned from beta-carotene and retinol efficacy trial, Cancer Epidemiology, Biomarkers \& Prevention 12 (2003), 518-526.

[63] E. Giovannucci, E.B. Rimm, Y. Liu, M. Leitzmann, K. Wu, M.J. Stampfer and W.C. Willett, Body mass index and risk of prostate cancer in U.S. health professionals, Journal of the National Cancer Institute 95 (2003), 1240-1244.

[64] I. Rusyn, S. Asakura, B. Pachkowski, B.U. Bradford, M.F. Denissenko, J.M. Peters, S.M. Holland, J.K. Reddy, M.L. Cunningham and J.A. Swenberg, Expression of Base Excision DNA Repair Genes Is a Sensitive Biomarker for in Vivo Detection of Chemical-induced Chronic Oxidative Stress: Identification of the Molecular Source of Radicals Responsible for DNA Damage by Peroxisome Proliferators, Cancer Res 64 (2004), 1050-1057.

[65] P. Lichtenstein, N.V. Holm, P.K. Verkasalo, A. Iliadou, J. Kaprio, M. Koskenvuo, E. Pukkala, A. Skytthe and K. Hemminki, Environmental and heritable factors in the causation of cancer: analyses of cohorts of twins from Sweden, Denmark and Finland, N Engl J Med 343 (2000), 78-85.
[66] J.P. Elo and T. Visakorpi, Molecular genetics of prostate cancer, Ann Med 33 (2001), 130-141.

[67] S.S. Padalecki, T.L. Johnson-Pais, A.M. Killary and R.J. Leach, Chromosome 18 suppresses the tumorigenicity of prostate cancer cells, Genes, Chromosomes \& Cancer 30 (2001), 221-229.

[68] G. Perinchery, M. Sasaki, A. Angan, V. Kumar, P. Carroll and R. Dahiya, Deletion of Y-chromosome specific genes in human prostate cancer, Journal of Urology 163 (2000), 1339-1342.

[69] J. Carpten, N. Nupponen, S. Issacs, R. Sood, C. Robbins, J. Xu, M. Faruque, T. Moses, C. Ewing, E. Gillanders, P. Hu, P. Bujnovszky, I. Makalowska, A. Baffoe-Bonnie, D. Faith, J. Smith, D. Stephan, K. Wiley, M. Brownstein, D. Gildea, B. Kelly, R. Jenkins, G. Hostetter, M. Matikainen, J. Schleutker, K. Klinger, T. Connors, Y. Xiang, Z. Wang, A. De Marzo, N. Papadopoulos, O.P. Kallioniemi, R. Burk, D. Meyers, H. Gronberg, P. Meltzer, R. Silverman, J. BaileyWilson, P. Walsh, W. Issacs and J. Trent, Germline mutations in the ribonuclease $\mathrm{L}$ gene in families showing linkage with HPC1, Nat Genet 30 (2002), 181-194.

[70] S.V. Tavtigian, J. Simard, D.H. Teng, V. Abtin, M. Baumgard, A. Beck, N.J. Camp, A.R. Carillo, Y. Chen, P. Dayananth, M. Desrochers, M. Dumont, J.M. Farnham, D. Frank, C. Frye, S. Ghaffary, J.S. Gupte, R. Hu, D. Iliev, T. Janecki, E.N. Kort, K.K. Laity, A. Leavitt, G. Leblanc, J. McArthurMorrison, A. Pederson, B. Penn, K.T. Peterson, J.E. Reid, S. Richards, M. Schroeder, R. Smith, S.C. Snyder, B. Swedlund, J. Swensen, A. Thomas, M.Tranchant, A.M. Woodland, F. Labrie, M.H. Skolnick, S. Neuhausen, J. Rommens and L.A. Cannon-Albright, A candidate prostate cancer susceptibility gene on 17p, Nat Genet 27 (2001), 172-180.

[71] D.C. Miller, S.L. Zheng, R.L. Dunn, A.V. Sarma, J.E. Montie, E.M. Lange, D.A. Meyers, J. Xu and K.A. Cooney, Germline mutations of the macrophage scavenger receptor 1 gene: association with prostate cancer risk in African-American men, Cancer Research 63 (2003), 3486-3489.

[72] J. Simard, M. Dumont, P. Soucy and F. Labrie, Perspective: prostate cancer susceptibility genes, Endocrinology 143 (2002), 2029-2040.

[73] T.R. Rebbeck, A.H. Walker, C. Zeigler-Johnson, S. Weisburg, A.M. Martin, K.L. Mathanson, A.J. Wein and S.B. Malkowicz, Am J Hum Genet 67 (2000), 1014-1019.

[74] J.P. Elo and T. Visakorpi, Molecular genetics of prostate cancer, Ann Med 33 (2001), 130-141.

[75] A. Balmain, J. Gray and B. Ponder, The gentics and genomics of cancer, Nature Genetics Suppl 33 (2003), 238-244.

[76] S.B. Gabriel et al., The structure of haplotype blocks in the human genome, Science 296 (2002), 2225-2229.

[77] L.R. Cardon and J.I. Bell, Association study designs for complex diseases, Nat. Rev. Genetics 2 (2001), 91-99.

[78] M. Verma and S. Srivastava, Epigenetics in cancer: implications for early detection and prevention, Lancet Oncology 3 (2002), 755-763.

[79] P.S. Rennie and C.C. Nelson, Epigenetic mechanisms for progression of prostate cancer, Cancer \& Metastasis Reviews 17(4) (1998-99), 401-409.

[80] W.G. Nelson, A.M. De Marzo, T.L. Deweese et al., Preneoplastic prostate lesions: an opportunity for prostate cancer prevention, Ann NY Acad Sci 952 (2001), 135-144.

[81] M. Yamanaka, M. Watanabe, Y. Yamada, A. Takagi, T. Murata, H. Takahashi, H. Suzuki, H. Ito, H. Tsukino, T. Katoh, Y. Sugimura and T. Shiraishi, Altered methylation of mul- 
tiple genes in carcinogenesis of the prostate, International Journal of Cancer 106 (2003), 382-387.

[82] S.V. Harden, H. Sanderson, S.N. Goodman, A.A. Partin, P.C. Walsh, J.I. Epstein and D. Sidransky, Quantitative GSTP1 methylation and the detection of prostate adenocarcinoma in sextant biopsies, Journal of the National Cancer Institute 95 (2003), 1634-1637.

[83] A.B. Jani and S. Hellman, Early prostate cancer: clinical decision-making, Lancet 361 (2003), 1045-1053.

[84] J.C. Presti, Prostate biopsy: how many cores are enough? Urologic Oncology: Seminars and Orig, Invest 21 (2003), 135-140.

[85] M.A. Khan, A.W. Partin, L.A. Mangold, J.I. Epstein and P.C. Walsh, Probability of biochemical recurrence by analysis of pathologic stage, Gleason score, and margin status for localized prostate cancer, Urology 62 (2003), 866-871.

[86] M.W. Kattan, S.F. Shariat, B. Andrews, K. Zhu, E. Canto, K. Matsumoto, M. Muramoto, P.T. Scardino, M. Ohori, T.M. Wheeler and K.M. Slawin, The addition of interleukin-6 soluble receptor and transforming growth factor beta 1 improves a preoperative nomogram for predicting biochemical progression in patients with clinically localized prostate cancer, Journal of Clinical Oncology 21 (2003), 3573-3579.

[87] M. Noguchi, T.A. Stamey, J.E. McNeal and R. Nolley, Prognostic factors for multifocal prostate cancer in radical prostatectomy specimens: lack of significance of secondary cancers, J Urol 170 (2003), 459-463.

[88] C.S. Foster, D.G. Bostwick, H. Bonkhoff, J.E. Damber, T. van der Kwast, R. Montironi and W.A. Sakr, Cellular and molecular pathology of prostate cancer precursors, Scand $J$ Urol Nephrol Suppl 205 (2000), 19-43.

[89] A. Villers, J.E. McNeal, F.S. Freiha and T.A. Stamey, Multiple cancers in the prostate. Morphologic features of clinically recognized versus incidental tumors, Cancer 70 (1992), 2313-2318.

[90] E.M. Tan, Autoantibodies as reporters identifying aberrant cellular mechanisms in tumorigenesis, J Clin Invest $\mathbf{1 0 8}$ (2001), 1411-1415.

[91] J.Y. Zhang, C.A. Casiano, X.X. Peng, J.A. Koziol, E.A. Chan and E.M. Tan, Enhancement of antibody detection in cancer using panel of recombinant tumor-associated antigens, Cancer Epidemiology, Biomarkers \& Prevention 12 (2003), 136-143.

[92] U. Sahin, O. Tureci, H. Schmitt, B. Cochlovius, T. Johannes, R. Schmits, F. Stenner, G. Luo, I.K. Schobert and M. Pfreundschuh, Human neoplasms elicit multiple specific immune responses in the autologous host, Proc Natl Acad Sci USA 92 (1995), 11810-11813.

[93] Y.T. Chen, M.J. Scanlan, Y. Obata and L.J. Old, Identification of human tumor antigens by serological expression cloning, in: Principles and practice of the biologic therapy of cancer, 3rd ed., S.A. Rosenberg, ed., Philadelphia: Lippincott
Williams and Wilkins, 2000, pp. 557-570.

[94] P.J. Mintz, J. Kim, K.A. Do, X. Wang, R.G. Zinner, M. Cristofanilli, M.A. Arap, W.K. Hong, P. Troncoso, C.J. Logothetis, R. Pasqualini and W. Arap, Fingerprinting the circulating repertoire of antibodies from cancer patients, Nature Biotechnology 21 (2003), 57-63.

[95] R.D. Unwin, P. Harnden. D. Pappin, D. Rahman, P. Whelan, R.A. Craven, P.J. Selby and R.E. Banks, Serological and proteomic evaluation of antibody responses in the identification of tumor antigens in renal cell carcinoma, Proteomics 3 (2003), 45-55.

[96] F. Brichory, D. Beer, F. Le Naour, T. Giordano and S. Hanash, Proteomics-based identification of protein gene product 9.5 as a tumor antigen that induces a humoral immune response in lung cancer, Cancer Research 61 (2001), 7908-7912

[97] J.C. Miller, H. Zhou, J. Kwekel, R. Cavallo, J. Burke, E.B. Butler, B.S. The and B.B. Haab, Antibody microarray profiling of human prostate cancer sera: antibody screening and identification of potential biomarkers, Proteomics 3 (2003), 56-63.

[98] T. Rabilloud, Membrane proteins ride shotgun, Nature Biotechnology 21 (2003), 508-510.

[99] M.B. Goshe, J. Blonder and R.D. Smith, Affinity labeling of highly hydrophobic integral membrane proteins for proteome-wide analysis, Journal of Proteome Research 2 (2003), 153-161.

[100] C.C. Wu, M.J. MacCoss, K.E. Howell and J.R. Yates 3rd, A method for the comprehensive proteomic analysis of membrane proteins, Nature Biotechnology 21 (2003), 532-538.

[101] T.F. Orntoft and E.M. Vestergaard, Clinical aspects of altered glycosylation of glycoproteins in cancer, Electrophoresis 20 (1999), 362-371.

[102] L. Xiong and F.E. Regnier, Use of lectin affinity selector in the search for unusual glycosylation in proteomics, J. Chromatog 782 (2002), 405-418.

[103] G. Chen, T.G. Gharib, H. Wang, C.C. Huang, R. Kuick, D.G. Thomasm, K.A. Shedden, D.E. Misek, J.M. Taylor, T.J. Giordano, S.L. Kardia, M.D. Iannettoni, J. Yee, P.J. Hogg, M.B. Orringer, S.M. Hanash and D.G. Beer, Protein profiles associated with survival in lung adenocarcinoma, Proceedings of the National Academy of Sciences of the United States of America 100 (2003), 13537-13542.

[104] J.D. Wulfkuhle, C.P. Paweletz, P.S. Steeg and E.F. Petricoin 3rd and L. Liotta, Proteomic approaches to the diagnosis, treatment, and monitoring of cancer, Advances in Experimental Medicine \& Biology 532 (2003), 59-68.

[105] D. Sidransky, Emerging molecular markers of cancer, Nat Rev Cancer 3 (2002), 210-219.

[106] M. Verma, J. Kagan, D. Sidransky and S. Srivastava, Proteomic analysis of cancer-cell mitochondria, Nat Rev Cancer 10 (2003), 789-795. 


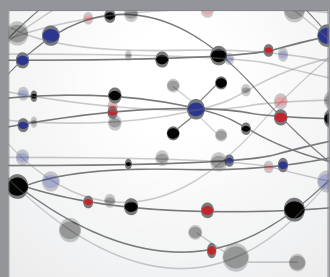

The Scientific World Journal
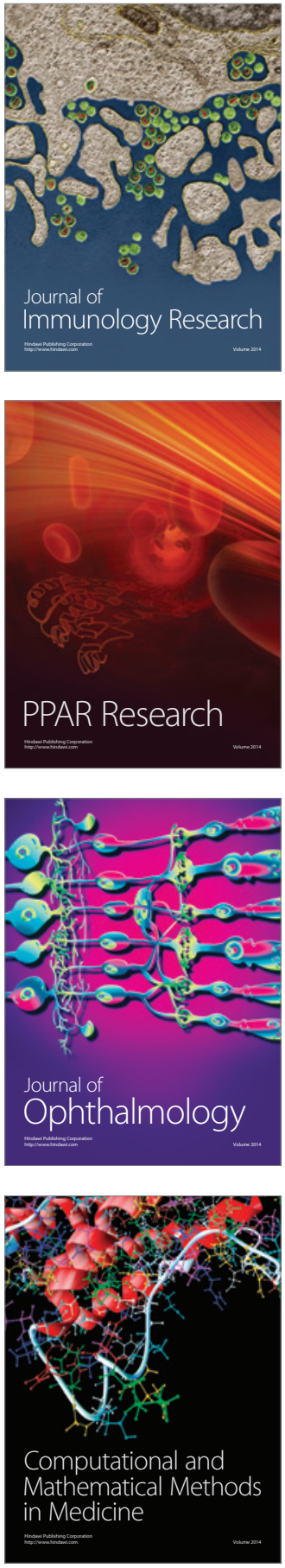

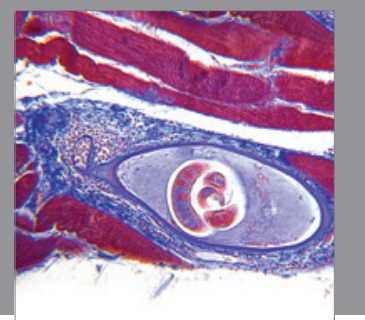

Gastroenterology

Research and Practice
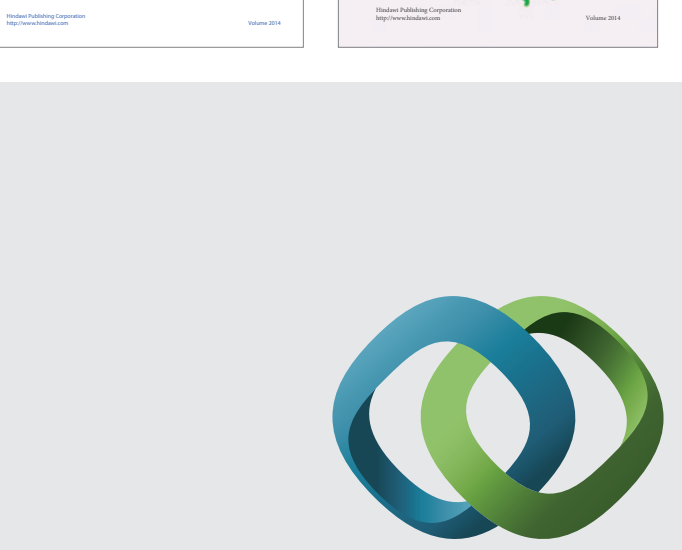

\section{Hindawi}

Submit your manuscripts at

http://www.hindawi.com
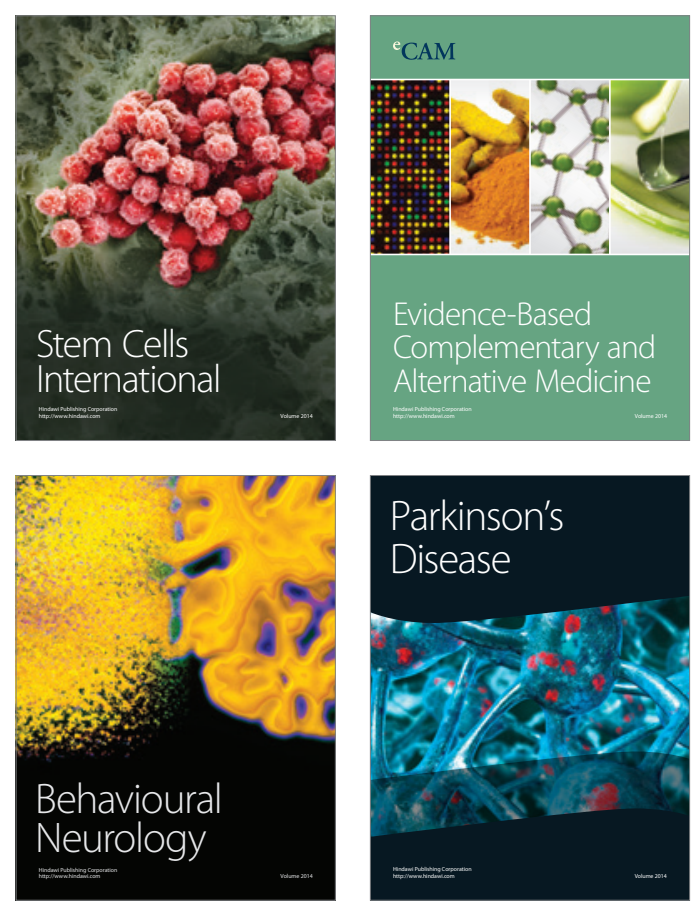

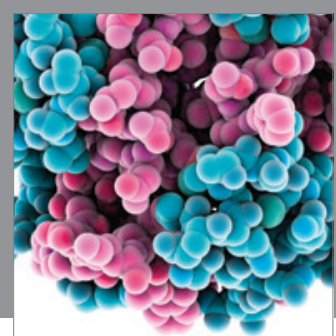

Journal of
Diabetes Research

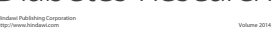

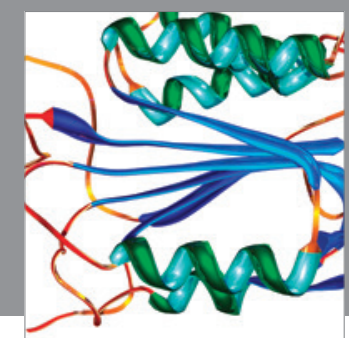

Disease Markers
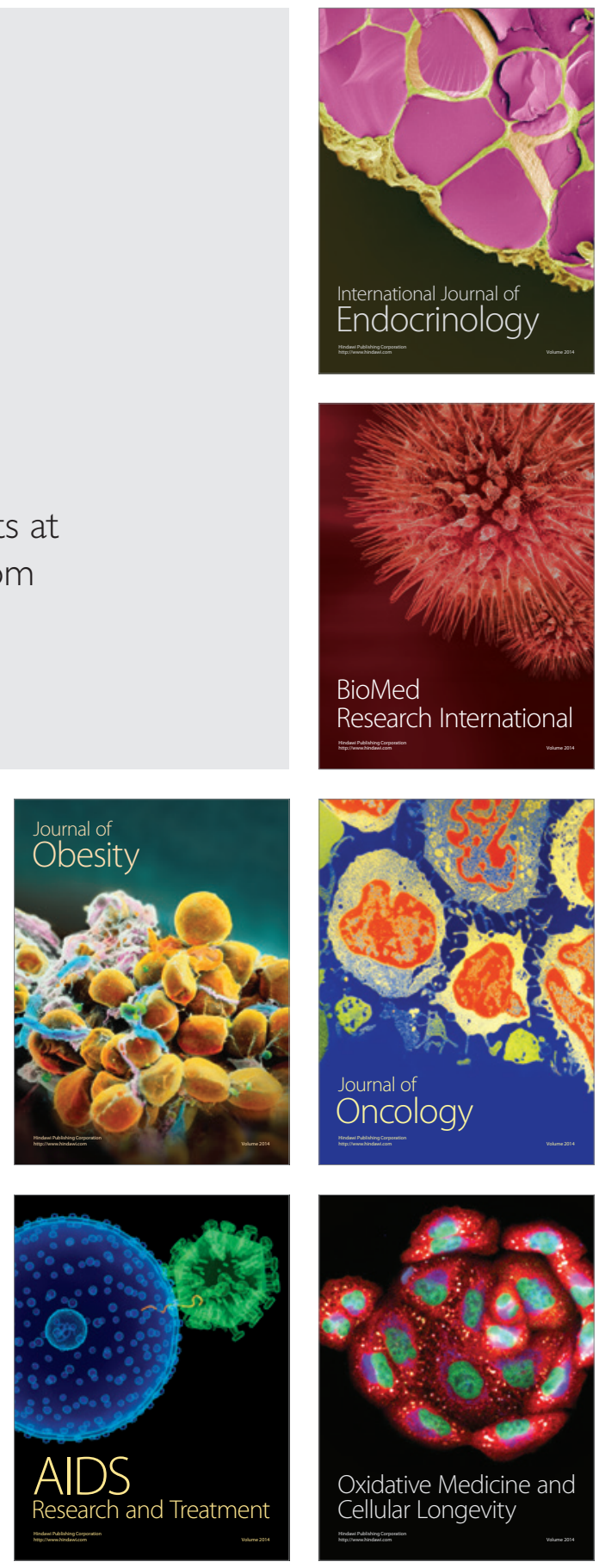